\title{
Hosing Instability in the Blow-Out Regime for Plasma-Wakefield Acceleration
}

\author{
C. Huang, ${ }^{1}$ W. Lu, ${ }^{1}$ M. Zhou, ${ }^{1}$ C. E. Clayton, ${ }^{1}$ C. Joshi, ${ }^{1}$ W. B. Mori, ${ }^{1}$ P. Muggli, ${ }^{2}$ S. Deng, ${ }^{2}$ E. Oz, ${ }^{2}$ T. Katsouleas, ${ }^{2}$ \\ M. J. Hogan, ${ }^{3}$ I. Blumenfeld, ${ }^{3}$ F. J. Decker, ${ }^{3}$ R. Ischebeck, ${ }^{3}$ R. H. Iverson, ${ }^{3}$ N. A. Kirby, ${ }^{3}$ and D. Walz ${ }^{3}$ \\ ${ }^{1}$ University of California, Los Angeles, California 90095, USA \\ ${ }^{2}$ University of Southern California, Los Angeles, California 90089, USA \\ ${ }^{3}$ Stanford Linear Accelerator Center, Menlo Park, California 94025, USA
}

\begin{abstract}
The electron hosing instability in the blow-out regime of plasma-wakefield acceleration is investigated using a linear perturbation theory about the electron blow-out trajectory in Lu et al. [in Phys. Rev. Lett. 96, 165002 (2006)]. The growth of the instability is found to be affected by the beam parameters unlike in the standard theory Whittum et al. [Phys. Rev. Lett. 67, 991 (1991)] which is strictly valid for preformed channels. Particle-in-cell simulations agree with this new theory, which predicts less hosing growth than found by the hosing theory of Whittum et al.
\end{abstract}

Recent experiments have shown amazing progress for both plasma-wakefield acceleration (PWFA) and laser wakefield acceleration (LWFA) [1-3] in the electron blow-out regime. In this regime, plasma electrons are completely evacuated by the space charge force of an electron beam or the ponderomotive force of a laser pulse, forming an ion channel on the axis of the system with a laminar sheath at the channel boundary carrying large concentrations of relativistic electrons. However, the electron hosing instability [4-7] of the drive and/or trailing beam remains a major concern for PWFA/LWFA concepts. The hosing instability results from the interaction between the electron sheath and the self-injected or externally injected electron beam. It leads to spatiotemporally growing oscillations of the beam centroid at each axial slice thus limiting the useful acceleration length and making it difficult to aim the beam. Existing standard theory $[4,6]$ predicts rapid growth for this instability. However, recent experiments $[1,2]$ have shown little evidence of hosing.

In this Letter, we present a more general hosing theory based on a perturbation method to the zeroth order trajectory [8] for the ion-channel/electron-sheath boundary. The initial hosing growth predicted by the linearized coupling is found to be affected by the nonconstant channel radius, relativistic mass corrections, and the longitudinal velocity of electrons in the plasma sheath. We verify this theory using particle-in-cell (PIC) simulations and compare it to the standard theory.

The existing work $[4,6]$ focused on the hosing in a long ion channel with a radius near the charge neutralization radius, i.e., $r_{c} \sim r_{\text {neu }}=\sqrt{n_{b} R_{b}^{2} / n_{p}}$, where $n_{b}, n_{p}$ are the beam and plasma density, respectively, and $R_{b}$ is the beam radius. Such a channel is either preformed or adiabatically formed. The electrons in the sheath layer are assumed to be at rest, i.e., the nonrelativistic limit; therefore, they do not generate or feel the magnetic fields. This adiabatic (referring to the channel formation), nonrelativistic (referring to the plasma sheath motion) limit is appropriate for a beam with a long bunch length $L \gg \lambda_{p}$ and weak charge per unit length $\Lambda=k_{p}^{2} r_{\text {neu }}^{2}=4 I_{b} / I_{A} \ll 1$, where $k_{p}=\omega_{p} / c$, $\omega_{p}=\sqrt{4 \pi n_{p} e^{2} / m}, \lambda_{p}=2 \pi k_{p}^{-1}$ are the wave number, angular frequency, and wavelength of the plasma wave moving at the speed of light, and $I_{b}$ and $I_{A}=17 \mathrm{kA}$ are the beam and Alfvén current, respectively. Such a beam creates a long channel with radius $r_{c} \approx r_{\text {neu }} \ll k_{p}^{-1}$; therefore, the plasma sheath is nonrelativistic. In this limit, the linearized coupled equations for the channel centroid $x_{c}$ and the beam centroid $x_{b}$ are [4,7]

$$
\partial_{s}^{2} x_{b}+k_{\beta}^{2} x_{b}=k_{\beta}^{2} x_{c}, \quad \partial_{\xi}^{2} x_{c}+\omega_{0}^{2} x_{c}=\omega_{0}^{2} x_{b},
$$

where $k_{\beta}=k_{p} / \sqrt{2 \gamma}, \omega_{0}=k_{p} / \sqrt{2}, s$ is the propagation distance into the plasma, $\xi=c t-z$ is the location within the beam, and $\gamma$ is the beam Lorentz factor. For a PWFA, the "short-pulse" limit of these equations is relevant, i.e., $k_{\beta} s \gg \omega_{0} \xi$, and the asymptotic solution for a linear tilt in $x_{b}$ is $x_{b} / x_{b 0}=0.341 A^{-3 / 2} e^{A} \cos \left(k_{\beta} s-A / \sqrt{3}+\pi / 4\right)$ [6], where $A=1.3\left[\left(k_{\beta} s\right)\left(\omega_{0} \xi\right)^{2}\right]^{1 / 3}$. In this short-pulse regime $\partial_{\xi}^{2} x_{c} \gg \omega_{0}^{2} x_{c}$ and $x_{b}$ oscillates nearly resonantly in $s$ with a wave number $k_{\beta}$.

In this Letter we investigate how the physics of the shortpulse asymptotic behavior is modified when the channel is formed nonadiabatically and when relativistic mass corrections arise (i.e., $L<\lambda_{p}, \Lambda>\sim 1$, and $r_{c, \max }>k_{p}^{-1}$ ). Under these conditions, the variation of $r_{c}$ along the beam is not negligible and the motion in the electron sheath can be relativistic. And using a fully explicit 3D simulation [9], hosing was found to be less severe than the standard theory prediction. The reasons for the reduced growth rate were not then clearly identified. Here we identify the physics that reduces the growth rate and quantify their effects.

We first illustrate how hosing occurs in the nonadiabatic, relativistic blow-out regime using a 3D fully nonlinear PIC code, QuickPIC [10], which uses the quasistatic approximation as discussed just after Eq. (2) below. Figure 1(a) 
shows the beam after the hosing instability has developed and saturated in the simulation. In Fig. 1(b) the centroid of the beam at the location of the left-hand (blue) arrow is plotted and compared to the asymptotic solution from the standard theory. The simulation parameters are as follows [9]: $n_{p}=2 \times 10^{16} \mathrm{~cm}^{-3}, n_{b} / n_{p}=25.9, k_{p} \sigma_{r}=0.19$, $k_{p} \sigma_{z}=1.2$, where $\sigma_{r}$ and $\sigma_{z}$ are beam RMS radius and length, respectively. The beam has an initial linear tilt of angle $\Theta=0.011$.

There are three regions along the beam which exhibit different behavior as indicated in Fig. 1(a) by the (yellow) box, left-hand (blue) arrow, and right-hand (green) arrow, respectively. In region I the ion channel has not yet completely formed and the latter part of the head gradually aligns with the front of the beam. In region II, the ion channel is fully formed and the electron sheath is laminar. Recently, Lu et al. [8] showed that the channel shape in this region can be represented by the trajectory of an electron at the channel boundary, defined as $r_{0}(\xi)$. We label the upper and lower trajectories as $r_{+}$and $r_{-}$, respectively. For a beam without a tilt, $r_{+}=r_{-}=r_{0}$. The linear (described by linearized equations) and nonlinear stages of hosing are seen in the lower red curve in Fig. 1(b) before and after $0.65 m$, respectively. In region III, the hosing amplitude becomes comparable to the channel radius and the beam breaks apart when it hits the channel boundary.

In this Letter, the linear stage of hosing growth in region II is studied. Clearly any theory for hosing of short pulses needs to include channel formation. (Our theory should also be valid when the plasma is created by self-ionization from the drive beam when the diameter of the ionized gas, $d \gg 2 r_{c}$. The opposite limit where $d \leq 2 r_{c}$ was studied in [11].) A perturbative model to the electron trajectory at the channel boundary is developed for small hosing amplitudes. To lowest order, the charge and current density perturbations at the boundary can be approximated as dipoles by a slightly shifted channel.

We use the coordinates $(x, y, s=z, \xi=c t-z)$ with $x z$ being the hosing plane and assume a quasistatic plasma response which essentially means that the $s$ dependence
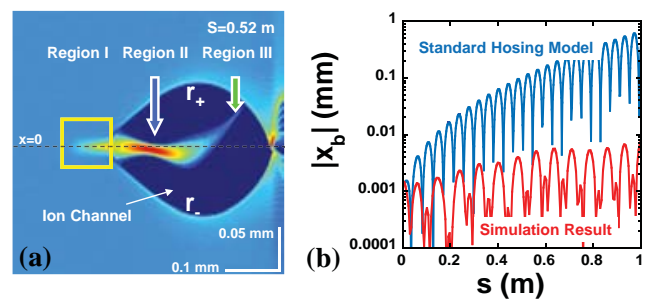

FIG. 1 (color). (a) A 2D slice of the beam (moving to the left) and plasma density after the beam has propagated $0.52 \mathrm{~m}$, illustrating the three regions of behavior. (b) The beam centroid $\left|x_{b}\right|$ (lower red curve) in the simulation (the asymmetry in this curve is due to beam centroid aligning to the axis at region I) and from the standard theory prediction (upper blue curve). The growth in the simulation is 1 order of magnitude smaller than the standard theory prediction. (except for that due to beam evolution) is removed when calculating the electron trajectory. The perturbation to the zeroth order trajectory is assumed to be small. Also, the focusing force is still assumed to be linear in $r$, i.e., channel deformation is omitted. The channel centroid can be defined as $x_{c}(\xi)=\left[r_{+}(\xi)-r_{-}(\xi)\right] / 2$. The channel diameter $d=r_{+}+r_{-}$is also assumed to be unchanged. Furthermore, the beam is assumed to be axisymmetric and narrow compared to $r_{0}$, so beam deformation can be neglected. Therefore, the coupling from the channel to the beam centroid $x_{b}$ is treated the same way as in [4].

Based on these assumptions, we derive a new equation for the channel centroid from the relativistic radial equation of motion for a plasma electron, beginning from what is essentially Eq. (3) of [8] but with no laser field

$$
\frac{d P_{\perp}}{d t}=\frac{d}{d t}\left(\gamma \frac{d r}{d t}\right)=-\left(E_{r}-V_{z} B_{\theta}\right)
$$

where $P_{\perp}, V_{z}$ are the perpendicular (radial) momentum and longitudinal velocity of a plasma electron, and $E_{r}$ and $B_{\theta}$ are the self-consistent radial electric field and azimuthal magnetic field, respectively. We normalize mass to $m$, charge to $e$, velocities to the speed of light $c$, lengths to $k_{p}^{-1}$, densities to $n_{p}$, and fields to $m c \omega_{p} / e$. Under the quasistatic approximation, $\gamma\left(1-V_{z}\right)=1+\psi$ [12], or $\gamma=\left[1+P_{\perp}^{2}+(1+\psi)^{2}\right] / 2(1+\psi)$, where the potential $\psi \equiv \phi-A_{z}$ is the solution to the Poisson equation $-\nabla_{\perp}^{2} \psi=4 \pi\left(\rho-J_{z}\right)$ in Lorentz gauge, and $\rho, J_{z}$ are plasma charge and longitudinal current densities, respectively. Thus $d / d t=\left(1-V_{z}\right) d / d \xi=(1+\psi) d /(\gamma d \xi)$ and $P_{\perp}=\gamma d r / d t=(1+\psi) d r / d \xi$. So Eq. (2) becomes (we define $d / d \xi \equiv^{\prime}$ )

$$
(1+\psi)\left[(1+\psi) r^{\prime}\right]^{\prime}=-\gamma\left(E_{r}-V_{z} B_{\theta}\right) .
$$

When the beam is straight, i.e., unperturbed, the solution to Eq. (3) is defined as $r_{0}(\xi)$ with other unperturbed quantities being $E_{0}, B_{0}, \psi_{0}, V_{0}(\xi)$, and $\gamma_{0}(\xi)$, respectively (subscripts $r, z$, and $\theta$ are dropped). Next, perturbations with subscript " 1 " are introduced.

$$
\begin{gathered}
r=r_{0}+r_{1}, \quad V=V_{0}+V_{1}, \\
E=E_{0}+E_{1}, \quad B=B_{0}+B_{1}, \\
\psi\left(r_{0}+r_{1}\right) \approx \psi_{0}\left(r_{0}\right), \text { i.e., } \psi_{1} \approx 0 .
\end{gathered}
$$

Equation (6) follows from the assumption that the channel is simply displaced by $r_{1}$ without deformation and the fact that $\psi$ is insensitive to the change in the profile of $\left(p-J_{z}\right)$ [8]. Equation (6), together with $\gamma=\left[1+P_{\perp}^{2}+\right.$ $\left.(1+\psi)^{2}\right] / 2(1+\psi)$ and $P_{\perp}=(1+\psi) r^{\prime}$, leads to $\gamma=$ $\gamma_{0}+\gamma_{1}=\gamma_{0}+\left(1+\psi_{0}\right) r_{0}^{\prime} r_{1}^{\prime}$. By substituting $\gamma$ and Eqs. (4)-(6) into Eq. (3) and ordering the resulting terms, we obtain the zeroth order Eq. (7) and first order Eq. (8),

$$
\left(1+\psi_{0}\right)\left[\left(1+\psi_{0}\right) r_{0}^{\prime \prime}+\psi_{0}^{\prime} r_{0}^{\prime}\right]=-\gamma_{0}\left(E_{0}-V_{0} B_{0}\right)
$$


TABLE I. Simulation parameters, and $\gamma=55,773$ for each case.

\begin{tabular}{ccccc}
\hline \hline Parameter & Simulation (a) & Simulation (b) & Simulation (c) & Simulation (d) \\
\hline$n_{p}\left(10^{16} \mathrm{~cm}^{-3}\right)$ & 5.66 & 5.66 & 2.0 & 2.0 \\
$N_{b}\left(10^{10} e^{-}\right)$ & $0.02 / 0.12$ & $6 / 24$ & 0.018 & 0.9 \\
$\sigma_{r}\left(10^{-3} c / \omega_{p}\right)$ & 9.09 & 89.8 & 5.3 & 13 \\
$L\left(c / \omega_{p}\right)$ & $4.91 / 11.4$ & $4.91 / 8.72$ & 3.72 & 3.72 \\
$\Theta\left(10^{-3} \mathrm{rad}\right)$ & 1.96 & 7.69 & 4.17 & 4.17 \\
$r_{\text {blow-out }}\left(c / \omega_{p}\right)$ & 0.23 & 4.3 & 0.24 & 1.5 \\
$c_{\tau}$ & $\sim 1$ & $\sim 1$ & a vary $w / \xi$ & a vary $w / \xi$ \\
$c_{\psi}$ & $\sim 1$ & $<1$ & $\sim 1$ & $<1$ \\
\hline \hline
\end{tabular}

${ }^{a}$ See text.

$$
\left(1+\psi_{0}\right)\left[\psi_{0}^{\prime} r_{1}^{\prime}+\left(1+\psi_{0}\right) r_{1}^{\prime \prime}+\left(E_{0}-V_{0} B_{0}\right) r_{0}^{\prime} r_{1}^{\prime}\right]=-\gamma_{0}\left(E_{1}-V_{0} B_{1}-V_{1} B_{0}\right)
$$

After substituting the value for $\left(E_{0}-V_{0} B_{0}\right)$ from Eq. (7) into Eq. (8) and dividing by $\left(1+\psi_{0}\right)^{2}$, one finds

$$
r_{1}^{\prime \prime}+\left\{\psi_{0}^{\prime}\left(1+\psi_{0}\right)^{-1}-\gamma_{0}^{-1}\left[\left(1+\psi_{0}\right) r_{0}^{\prime \prime}+\psi_{0}^{\prime} r_{0}^{\prime}\right] r_{0}^{\prime}\right\} r_{1}^{\prime}=-\gamma_{0}\left(1+\psi_{0}\right)^{-2}\left(E_{1}-V_{0} B_{1}-V_{1} B_{0}\right) .
$$

Equation (9) is a second order ordinary differential equation for $r_{1}$ with coefficients depending on the zeroth order quantities and their $\xi$ derivatives. The first two terms in the last bracket on the right-hand side $-\left(E_{1}-V_{0} B_{1}\right)$ are the sum of the perturbation force from the displacement of the beam, $F_{b 1}$, and the change in the plasma self-force due to the displacement of the sheath, $F_{e 1}$. The former can be expressed as $F_{b 1}=-\left(E_{b 1}-V_{0} B_{b 1}\right)$, where $E_{b 1}$ and $B_{b 1}$ are the change to the electric and magnetic fields from the beam when the centroid is shifted by $x_{b}$, i.e.,

$$
E_{b 1}=B_{b 1}=-\frac{1}{2} n_{b} R_{b}^{2}\left[\left(r_{0}+r_{1}-x_{b}\right)^{-1}-r_{0}^{-1}\right] \text {. }
$$

Therefore, $\quad F_{b 1}=-\left(E_{b 1}-V_{0} B_{b 1}\right) \approx \frac{1}{2} n_{b} R_{b}^{2}\left(1-V_{0}\right) \times$ $\left(x_{b}-r_{1}\right) / r_{0}^{2}$ when $\left|x_{b}-r_{1}\right| \ll r_{0}$. Evaluating $F_{e 1}$ from the actual displacement of the channel would be more complicated. We argue, however, that to lowest order it can be neglected. Under the assumption (confirmed by simulation) that the channel shape does not change significantly then $\phi_{1} \approx A_{z 1} \approx 0$; thus $F_{e 1}=E_{x 1}-V_{0} B_{y 1}=$ $-\left(1-V_{0}\right) \partial_{\xi} A_{x 1}$, where $A_{x 1}$ is the perturbation to the $x$ component of the vector potential, and $A_{x 1}$ satisfies $-\nabla_{\perp}^{2} A_{x 1}=4 \pi J_{x 1}$ with $J_{x 1}$ being a first order term in $r_{1}$. To lowest order, $A_{x 1}(y=0) \approx f\left[r_{0}(\xi)\right] r_{1} V_{x 0}$, where $V_{x 0}$ is the magnitude of the velocity in the $\hat{x}$ direction for an electron at the channel boundary. $F_{e 1}=-\left(1-V_{0}\right) \partial_{\xi} A_{x 1}$ can now be neglected because $V_{x 0} \ll 1$ in much of the channel and because we are interested in the "short pulse" for which $\partial_{\xi} r_{0} / r_{0} \approx 1 / r_{0} \ll \partial_{\xi} r_{1} / r_{1}$.

The last term $V_{1} B_{0}$ in Eq. (9) can be expressed in terms of $r_{1}$ by noting that if $\psi_{1}=0$ then $V_{1}=\gamma_{1}\left(1-V_{0}\right) \gamma_{0}^{-1}$, $\gamma_{1}=\left(1+\psi_{0}\right) r_{0}^{\prime} r_{1}^{\prime}$, and $B_{0}=-\frac{1}{2} n_{b} R_{b}^{2} r_{0}^{-1}=-\frac{1}{2} \Lambda r_{0}^{-1}$, giving $V_{1} B_{0}=-\frac{\Lambda}{2} r_{0}^{-1}\left(1+\psi_{0}\right)^{2} \gamma_{0}^{-2} r_{0}^{\prime} r_{1}^{\prime}$ which is proportional to $r_{1}^{\prime}$. Next we drop all the $r_{1}^{\prime}$ terms in Eq. (9) because $\psi_{0}^{\prime}, \psi_{0}^{\prime} / \psi_{0}, r_{0}^{\prime \prime}, r_{0}^{\prime} / r_{0}^{\prime} \ll r_{1}^{\prime \prime} / r_{1}^{\prime}$ in the "short-pulse" limit. Therefore, Eq. (9) can be written as

$$
r_{1}^{\prime \prime}+c_{r} c_{\psi} \omega_{0}^{2} r_{1}=c_{r} c_{\psi} \omega_{0}^{2} x_{b}
$$

where $c_{r} \equiv n_{b} R_{b}^{2} / r_{0}^{2}=r_{\text {neu }}^{2} / r_{0}^{2}$ and $c_{\psi} \equiv 1 /\left(1+\psi_{0}\right)=$ $1 /\left[\gamma_{0}\left(1-V_{0}\right)\right]$ now both vary along the trajectory. Equation (11) is for the upper trajectory $r_{+}=r_{0}+r_{1}$. For the lower trajectory $r_{-}=r_{0}+r_{2}, r_{2}$ satisfies a similar equation. So $x_{c}=\left(r_{+}-r_{-}\right) / 2=\left(r_{1}-r_{2}\right) / 2$ satisfies

$$
x_{c}^{\prime \prime}+c_{r} c_{\psi} \omega_{0}^{2} x_{c}=c_{r} c_{\psi} \omega_{0}^{2} x_{b} .
$$

The equation for beam centroid is the same as Eq. (1).

The differences between Eq. (12) and (1) are $c_{r}, c_{\psi}$. In the adiabatic nonrelativistic limit, $V_{x 0} \approx 0, r_{0} \approx r_{\text {neu }}$, $V_{0} \ll 1$; hence $c_{r} \approx 1, \psi_{0} \approx 0$, and $c_{\psi} \approx 1$. Therefore, we recover the result in [4], i.e., Eq. (1). For more intense beam-plasma interactions: (1) the channel radius varies along the beam; (2) the relativistic mass changes the plasma electron resonant frequency and the magnetic field is important due to large $V_{0}$. These two effects change the coefficients $c_{r}$ and $c_{\psi}$, respectively, thereby changing the
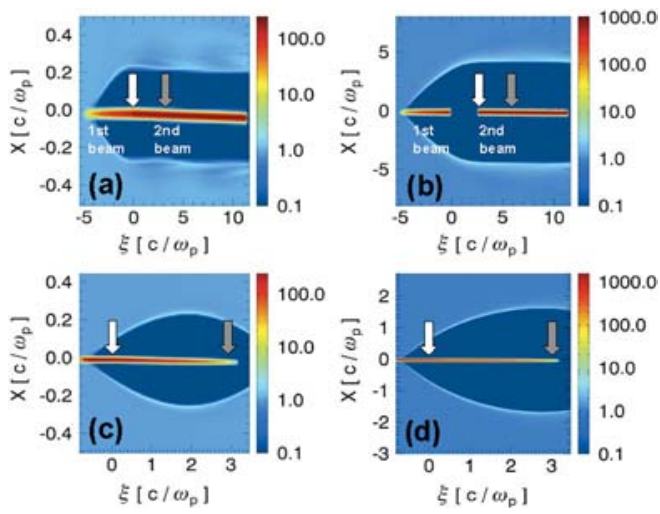

FIG. 2 (color). The beams (red, moving to the left) and the plasma (blue) in (a) the adiabatic nonrelativistic regime, (b) the adiabatic relativistic regime, (c) the nonadiabatic nonrelativistic regime, (d) the nonadiabatic relativistic regime. Left-hand (white) and right-hand (gray) arrows denote where tilts are added and the centroids are measured. 


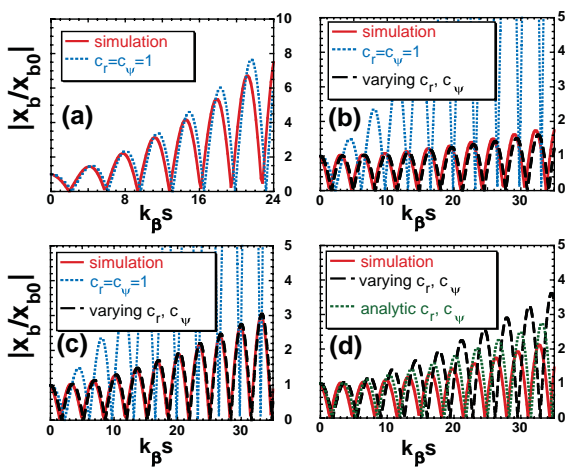

FIG. 3 (color). Hosing growth in four regimes. $x_{b 0}$ is the initial displacement of the beam centroid. We assume $\Delta_{s}=0.1 r_{0}$, $\Delta_{L}=1 c / \omega_{p}$ for the analytic curve in case (d). The slightly slower hosing growth in simulation (d) is caused by nonlinearity in beam-channel centroid coupling.

hosing growth. Generally, $c_{r} c_{\psi}<1$ in a nonadiabatic relativistic channel, therefore reducing hosing growth.

To verify this new hosing theory and to study the effects of $c_{r}$ and $c_{\psi}$, we conduct QuickPIC simulations in the (a) adiabatic nonrelativistic regime, (b) the adiabatic relativistic regime, (c) the nonadiabatic nonrelativistic regime, and (d) the nonadiabatic relativistic regime, respectively. Table I summarizes the parameters and the roles of $c_{r}$ and $c_{\psi}$ and Fig. 2 shows the initial beam and the shape of the channel in these simulations. In case (a) and (b), a nonevolving beam is used to create an initial channel, while the trailing beam propagates in an adiabatic (long flat) channel with $r_{0} \approx r_{\text {neu }}$ and $c_{r} \approx 1$. In case (c) and (d), a beam with a triangular longitudinal profile creates its own channel. The beam density reaches its peak at $0.27 c / \omega_{p}$ from the head so the ion channel radius varies significantly (nonadiabatically) along the beam but for most part $r_{0}>r_{\text {neu }}$ and $c_{r}<1$. Futhermore, the beams in case (b) and (d) have more charge than in case (a) and (c), resulting in relativistic channels with larger $r_{0}$ and $c_{\psi}<1$ (see Table I). To trigger the hosing instability, a small linear tilt with angle $\Theta$ is imposed on the second beam for case (a) and (b) and at $0.8 c / \omega_{p}$ for case (c) and (d), with the head of the beam on axis and the tail off axis. The beam centroid oscillations at $3 c / \omega_{p}$ behind from where the tilts are added are plotted as solid red curves in Fig. 3 for all four regimes.

We take zeroth order quantities from simulations and numerically integrate Eq. (12) and the beam equation from (1). The results [dashed black curves in Figs. 3(b)-3(d)] agree well with the simulations, while the standard theory [dotted blue curves labeled $c_{r}=c_{\psi}=1$ in Fig. 3, not shown in 3(d)] overestimates the hosing by 1 order of magnitude except for case (a). Therefore, the relatively low hosing growth rate is no longer an anomaly, and our simple model can now be used with confidence to discuss future projects.

In [8] an approximate but analytic model, resulting in Eqs. (10), (11) of [8], was created and used. Using an approximate sheath layer width $\Delta_{s}$ and linear response layer width $\Delta_{L}$, this model relates $\psi$ to $r_{0}$, involving a complicated parameter $\beta\left(r_{0}, \Delta_{s}, \Delta_{L}\right)$ [as defined between Eqs. (10), (11) of [8]]. From this model one also has the useful result $\psi_{0}=\beta r_{0}^{2} / 4$; thus $c_{\psi}=1 /\left(1+\beta r_{0}^{2} / 4\right)$ is small for large $r_{0}$, i.e., the relativistic limit. This model can also be used to directly calculate $c_{r}$ and $c_{\psi}$ rather than going through simulation. The result [dotted green curve labeled as "analytic" in Fig. 3(d)] works well comparing with the simulation.

Assuming constant $c_{r}$ and $c_{\psi}$, our theory predicts $x_{b} / x_{b 0}$ has the same asymptotic expression in the "short-pulse" limit as the standard theory result but with $A=$ $1.3\left[c_{r} c_{\psi}\left(k_{\beta} s\right)\left(\omega_{0} \xi\right)^{2}\right]^{1 / 3}$. Clearly, reducing the bunch length is still the most effective way to suppress hosing, while the nonadiabatic relativistic regime is advantageous because typically $c_{r} c_{\psi}=r_{\text {neu }}^{2} r_{0}^{-2}\left(1+\beta r_{0}^{2} / 4\right)^{-1} \sim O(0.1)$. Therefore, the propagation distance can be made about 10 times longer for the same tolerance of hosing growth. In the recent E167 experiment [2], the beam had $N_{b}=1.8 \times$ $10^{10}$ electrons, spot sizes of $\sigma_{r}=10 \mu \mathrm{m}$, a bunch length (in $\xi$ ) of $\sigma_{z}=15 \mu \mathrm{m}$, and $\gamma_{b}=82,192 . k_{\beta} s=205$ for $85 \mathrm{~cm}$ long plasma at $n_{p}=2.7 \times 10^{17} \mathrm{~cm}^{-3}$. Assuming a constant blow-out radius, we obtain $c_{r} \approx 0.25, c_{\psi} \approx 0.54$. Thus $A$ is reduced from 7.7 to 3.95 and $x_{b} / x_{b 0}$ is reduced from 35 to only 2.3 for $\omega_{0} \xi=1$ [13].

Last, our theory indicates that the hosing growth rate depends on the beam parameters. When $I_{b}$ increases, $c_{r}$ and $c_{\psi}$ both decrease, thereby reducing hosing growth. Furthermore, the hosing amplitude is not amplified between the drive/trailing beams where $c_{r}=0$. These conclusions may help designing more stable PWFA experiments.

Work was supported by DOE under Contracts No. DEFC02-01ER41179, No. DE-FG02-03ER54721, No. DEFG03-92-ER40727, No. DE-FG02-03NA00065, No. DEFG02-97ER41039, No. DE-FG02-92ER40745, and No. DE-FC02-01ER41192, and by NSF under Grant No. PHY0317271. Simulations were performed at local Dawson cluster and NERSC.

[1] M. Hogan et al., Phys. Rev. Lett. 95, 054802 (2005).

[2] I. Blumenfeld et al., Nature (London) 445, 741 (2007).

[3] W. P. Leemans et al., Nature Phys. 2, 696 (2006).

[4] D. H. Whittum et al., Phys. Rev. Lett. 67, 991 (1991).

[5] J. Krall et al., Phys. Plasmas 2, 1326 (1995).

[6] A. A. Geraci et al., Phys. Plasmas 7, 3431 (2000).

[7] M. Lampe et al., Phys. Fluids B 5, 1888 (1993).

[8] W. Lu et al., Phys. Rev. Lett. 96, 165002 (2006).

[9] E. S. Dodd et al., Phys. Rev. Lett. 88, 125001 (2002).

[10] C. Huang et al., J. Comp. Phys. 217, 658 (2006).

[11] S. Deng et al., Phys. Rev. Lett. 96, 045001 (2006).

[12] P. Mora et al., Phys. Plasmas 4, 217 (1997).

[13] In the experiment, $\sigma_{r}$ is comparable to $r_{c}$, which further reduced the growth rate. See [2]. 